\title{
Anisotropy in high-frequency broadband acoustic backscattering in the presence of turbulent microstructure and zooplankton
}

\author{
Doris Leong $^{\text {a) }}$ and Tetjana Ross \\ Department of Oceanography, Dalhousie University, Halifax, Nova Scotia B3H 4J1, Canada \\ Andone Lavery \\ Woods Hole Oceanographic Institution, Woods Hole, Massachusetts 02543
}

(Received 6 August 2010; revised 1 June 2012; accepted 1 June 2012)

\begin{abstract}
High-frequency broadband $(120-600 \mathrm{kHz})$ acoustic backscattering measurements have been made in the vicinity of energetic internal waves. The transducers on the backscattering system could be adjusted so as to insonify the water-column either vertically or horizontally. The broadband capabilities of the system allowed spectral classification of the backscattering. The distribution of spectral shapes is significantly different for scattering measurements made with the transducers oriented horizontally versus vertically, indicating that scattering anisotropy is present. However, the scattering anisotropy could not be unequivocally explained by either turbulent microstructure or zooplankton, the two primary sources of scattering expected in internal waves. Daytime net samples indicate a predominance of short-aspectratio zooplankton. Using zooplankton acoustic scattering models, a preferential orientation of the observed zooplankton cannot explain the measured anisotropy. Yet model predictions of scattering from anisotropic turbulent microstructure, with inputs from coincident microstructure measurements, were not consistent with the observations. Possible explanations include bandwidth limitations that result in many spectra that cannot be unambiguously attributed to turbulence or zooplankton based on spectral shape. Extending the acoustic bandwidth to cover the range from $50 \mathrm{kHz}$ to $2 \mathrm{MHz}$ could help improve identification of the dominant sources of backscattering anisotropy.

(C) 2012 Acoustical Society of America. [http://dx.doi.org/10.1121/1.4730904]
\end{abstract}

PACS number(s): 43.30.Ft, 43.30.Pc [FGF]

Pages: 670-679

\section{INTRODUCTION}

High-frequency acoustic backscattering methods have proven to be an extremely useful oceanographic tool, as they can provide high-resolution images of the distribution of zooplankton and oceanic microstructure over large spatial scales (e.g., Haury et al., 1983; Sandstrom and Oakey, 1995; Wiebe et al., 1997; Moum et al., 2003). Taking backscattering data beyond imaging and attempting to produce quantitative measures of turbulence parameters and plankton biomass has been the subject of considerable research (e.g., Holliday and Pieper, 1995; Ross and Lueck, 2005; Lavery et al., 2010a). Though narrowband acoustic scattering techniques have been the focus of much of the research to date, broadband techniques are increasingly being applied as they allow improved spectral classification of the different sources of scattering (e.g., Stanton et al., 1994; Lavery et al., 2010a,b; Stanton et al., 2010). Scattering anisotropy, where measurements in different directions do not yield the same result, complicates the interpretation of acoustic images, even when broadband acoustic spectra are available. Though accounting for anisotropy is important to the accurate interpretation of observed high-frequency backscattering, there have only been a few studies that have directly examined volume scattering anisotropy in the field.

\footnotetext{
a) Author to whom correspondence should be addressed. Electronic mail: doris.leong@geog.ubc.ca
}

This study focuses on broadband measurements, spanning a frequency range from $120 \mathrm{kHz}$ to $600 \mathrm{kHz}$, of scattering anisotropy in the presence of internal waves. The two primary high-frequency sources of scattering expected in internal waves are zooplankton and turbulent oceanic microstructure. There are extensive laboratory scattering measurements using both narrowband and broadband acoustic scattering techniques, of the angular dependence of scattering from elongated zooplankton (e.g., Martin Traykovski, 1998; Warren et al., 2002; Roberts and Jaffe, 2008), supported by extensive scattering models of varying complexity (e.g., Stanton and Chu, 2000; Simmonds and MacLennan, 2005). For example, Roberts and Jaffe (2008) found that a mysid $(8-12 \mathrm{~mm}$ in length) and a copepod $(1-4 \mathrm{~mm}$ in length) can have very similar spectra and may be indistinguishable depending on the angle of insonification. These laboratory studies are supported by in situ studies (e.g., Trevorrow et al., 2005; Lawson et al., 2006; Wiebe et al., 2010), which have corroborated that animal orientation can significantly affect acoustic scattering levels, particularly for zooplankton with large aspect ratios. However, there are few focused, in situ measurements of scattering anisotropy due to zooplankton orientation (Sameoto, 1980; Jaffe et al., 1998). Anisotropic backscattering by zooplankton may arise from preferential animal orientation in response to various external or internal stimuli (Naylor, 2006). If whole populations are oriented primarily in one direction, this may contribute to differences between horizontally and vertically sampled scattering because zooplankton can scatter more 
strongly if observed from broadside than from end-on incidence (McGehee et al., 1998). As a result, recent models take into account a distribution of animal orientations (e.g., Lavery et al., 2007; Simard and Sourisseau, 2009), which have been observed for some common bioacoustic taxa (Midttun, 1984). Certain copepods were found to preferentially orient vertically (Benfield et al., 2000), while pteropods tend to orient their opercular opening upward, giving them a stronger mean target strength when observed horizontally (Warren et al., 2002). Euphausiids have been observed to preferentially orient horizontally (Sameoto, 1980; Lawson et al., 2006), but in some cases also orient $10^{\circ}-20^{\circ}$ from the horizontal (Chu et al., 1993). Adding uncertainty to these results is evidence that euphausiids have different distributions of orientations at different times of day (Sameoto, 1980; Simard and Sourisseau, 2009). While models are ready to incorporate zooplankton orientation for some species, there are insufficient in situ observations for many other species to constrain the models. Assessing scattering anisotropy is even more complicated in this experiment because little is known about the influence of internal waves on the preferential orientation of zooplankton.

In contrast to zooplankton, there are few laboratory measurements of scattering from turbulent microstructure, for example the series of laboratory measurements culminating in Oeschger and Goodman (2003). Traditionally, it is assumed that turbulent microstructure is isotropic at small scales (Kolmogorov, 1941; Monin and Yaglom, 1965; Thorpe, 2005; Moum and Rippeth, 2009) and scattering models for turbulent microstructure make the same simplifying assumption (Seim, 1999; Lavery et al., 2003; Ross and Lueck, 2005). Scattering models for turbulent microstructure based on isotropy have evolved considerably in sophistication in the last decade: Goodman (1990) addressed scattering from turbulent temperature microstructure, Seim (1999) added salinity, Lavery et al. (2003) added density, and Ross et al. (2004) introduced a new form for the temperature-salinity cross-spectrum. Direct observations of turbulence anisotropy by Gargett (1984), however, have shown that turbulence becomes increasingly anisotropic as the buoyancy Reynolds number decreases. The assumption of isotropy, along with measurements made at a single angle of insonification, may therefore lead to acoustic inversions that incorrectly estimate important turbulence parameters, such as turbulent dissipation rates. Some progress has been made toward developing acoustic backscattering models that include the effects of anisotropic turbulence (Goodman, 1990), however, the importance of scattering anisotropy due to turbulent oceanic microstructure remains, for the most part, an open question.

Although the directional dependence of backscattering from both microstructure and zooplankton may be crucial to the accurate interpretation of data collected at different angles of insonification, much remains to be done before it can be properly accounted for. Typically, high-frequency acoustic observations are made with the transducers oriented vertically, either looking down from a ship or up from a mooring. Increasingly, techniques are being improved to provide measurements made at other angles, either side-looking monostatic systems (e.g., Ross and Lueck, 2005; Sutor et al., 2005) or multi-beam systems with a range of angles of insonification (e.g., Bertrand et al., 2008; Benoit-Bird and Au, 2009). Models that accurately parameterize anisotropy in backscattering from turbulent microstructure and zooplankton are an important ingredient, as are direct measurements of scattering anisotropy. This study examines backscattering from turbulent and zooplankton sources in nonlinear internal solitary waves using a bi-directional, high-frequency broadband acoustic system. Section II describes the instruments and methods of data collection and Sec. III discusses the classification scheme for sorting spectra according to their shape. Section IV presents a statistical comparison between two acoustic sampling directions. Sections V and VI consider zooplankton and turbulence anisotropy in turn, and Sec. VII presents some concluding remarks and suggestions for future work.

\section{EXPERIMENT}

Observational data were collected on board the $R / V$ Oceanus along the New Jersey continental shelf during a wave-tracking experiment in coordination with the SW06 Shallow Water Acoustics Experiment (Tang et al., 2007). During a month-long cruise in August 2006, trains of nonlinear solitary internal waves of depression, occurring in the highly stratified shelf waters, were tracked and sampled repeatedly using a broadband acoustic system. Coincident direct microstructure measurements were performed, as well as occasional zooplankton net tows (see Lavery et al., 2010a,b for more details). Figure 1 shows a map of the region and indicates the sampling paths and locations of the different internal wave trains included in this study.

\section{A. Broadband acoustic sampling}

The broadband acoustic instrument, detailed in Lavery et al. (2010a), is a monostatic system that measures frequency

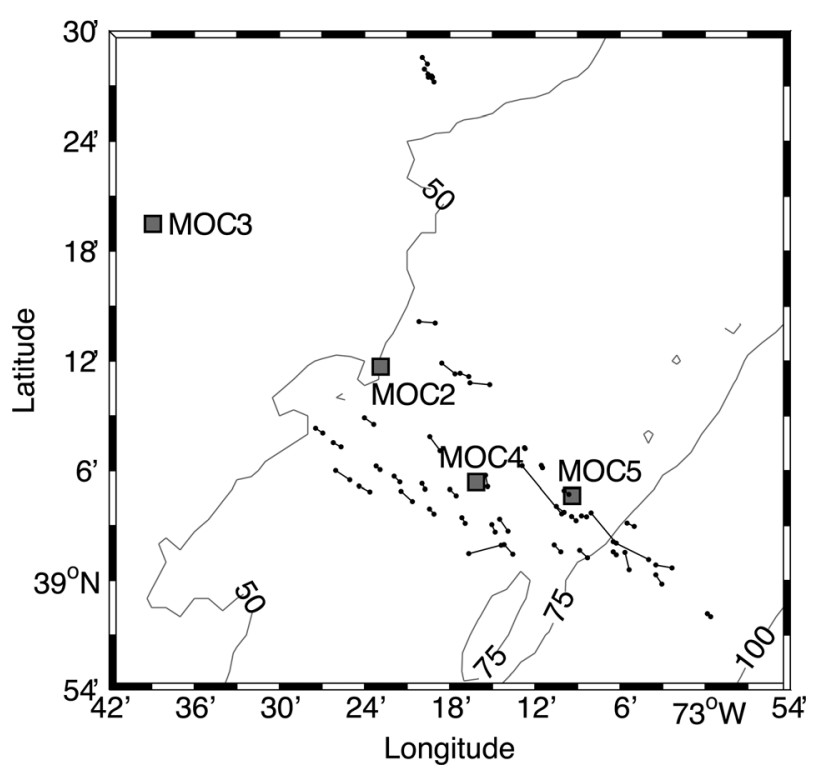

FIG. 1. Location of observations on the New Jersey continental shelf. The bathymetry of the region is shown with contour lines of depth in units of meters. The path of each internal wave included in this study is shown between the endpoints of each line, and the location of MOCNESS net tows are labeled with squares. 


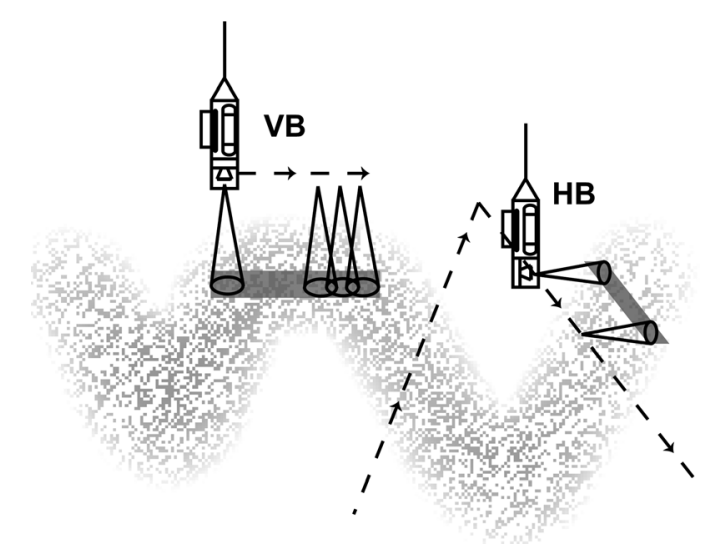

FIG. 2. Diagram of sampling geometry for both instrument orientations used during the data collection. When sampling vertically, the instrument hung at a constant depth, facing down. In this orientation, the sampling volume height was determined by the selected range over which spectral averaging was performed, while the number of pings averaged and the frequency dependent beam width determined the effective sampling volume width. When sampling horizontally, the instrument was usually profiled vertically. In this orientation, both the effective sampling volume height and width were a function of the range, number of pings, and also the profiling speed of the instrument.

dependent backscattering over four frequency bands-LOW: 160-270 kHz, MID: $220-330 \mathrm{kHz}, \mathrm{HL}: 330-470 \mathrm{kHz}, \mathrm{HH}$ : $450-590 \mathrm{kHz}$. The LOW and MID bands have a ping rate of $1 \mathrm{~Hz}$, while the $\mathrm{HL}$ and $\mathrm{HH}$ bands ping at half that rate. To address backscattering anisotropy, the transducers were mounted on a rotatable plate that can be oriented to horizontally or vertically insonify the water column. The instrument was deployed over the ship's starboard side as the ship moved toward the approaching wave train at approximately $0.5 \mathrm{~ms}^{-1}$. The instrument operated in one of two sampling orientations during each instrument cast (Fig. 2). In the vertical backscattering (VB) mode, the instrument was maintained at a fixed depth, with the transducers downward-looking into the watercolumn. For the horizontal backscattering (HB) mode, the instrument was profiled vertically through the water column, with the transducers looking horizontally. A stabilizing fin was incorporated into the design of the instrument to orient the instrument into the approaching wave train.

The received backscattering was processed using pulsecompression techniques, which improve the signal-to-noise ratio and range resolution of the instrument (Chu and Stanton, 1998; Stanton and Chu, 2008). The system was calibrated using the standard target approach discussed in Lavery et al. (2010a) The acoustic spectra represent volume scattering strength $\left(S_{v}\right.$, where $S_{v}=10 \log _{10} \sigma_{\mathrm{bs}}$ and $\sigma_{\mathrm{bs}}$ is the mean backscattering cross-section per unit volume) in decibels relative to $1 \mathrm{~m}^{-1}$ $\left(\mathrm{dB}\right.$ re $\left.\mathrm{m}^{-1}\right)$, as a function of acoustic frequency. Pulse lengths of $5 \mathrm{~ms}$ and $500 \mu \mathrm{s}$ were used for the VB and HB modes, respectively. This results in the VB signal having a higher signal-to-noise ratio, but a larger blanking range. Noise levels were in the range of -90 to $-85 \mathrm{~dB}$ for the LOW and MID bands and between -75 to $-70 \mathrm{~dB}$ for the $\mathrm{HL}$ and $\mathrm{HH}$ bands.

Typical temperature and salinity profiles for the New Jersey shelf are shown in Fig. 3(a). An image of compressed pulse output in the LOW band and VB mode shows a passing internal wave train in Fig. 3(b). The density contours, calculated from coincident direct microstructure data (Sec. II B), show a pycnocline that is aligned with a clear mid-depth
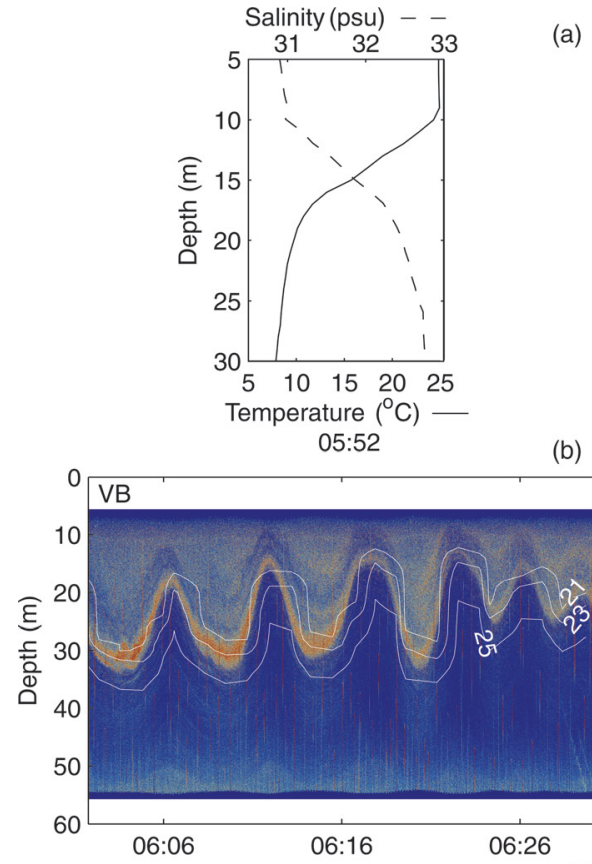

(a)

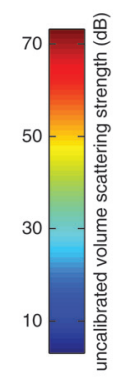

(b)

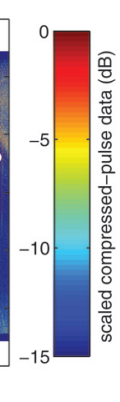

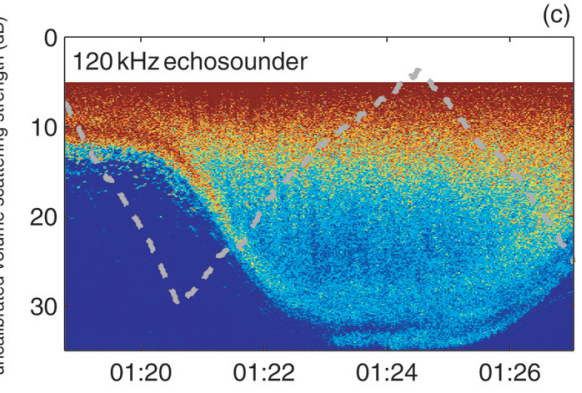

(c)

(d)

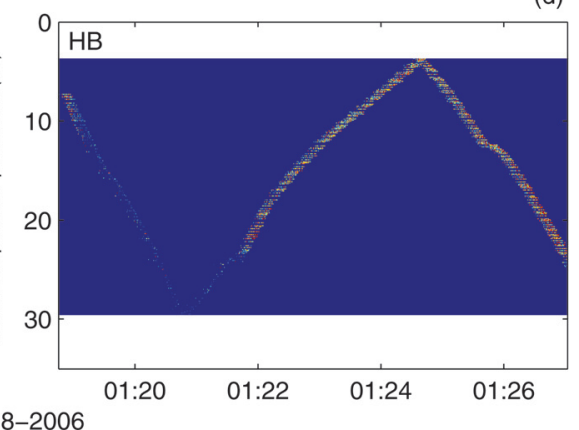

FIG. 3. (a) Typical temperature and salinity profiles along the New Jersey continental shelf. (b) Compressed pulse output, from the VB LOW band, showing a passing internal wave on August 18, $2006(06: 00-06: 30) . \sigma\left(\rho-1000 \mathrm{kgm}^{-3}\right)$ contours, calculated from Chameleon microstructure data, show that the pycnocline occurs along the interface of internal wave propagation. The instrument was hanging at a fixed depth near the surface, looking vertically downward. (c) $120 \mathrm{kHz}$ ship-mounted echosounder image of a passing internal wave train on August 18, 2006 (01:21-01:27). The track of the broadband instrument while in HB mode is overlaid. (d) Compressed pulse output from the HB LOW band, showing scattering up to a range of $25 \mathrm{~m}$ and coincident in time with the above panel. Note that the pattern of scattering in the broadband acoustics matches the general pattern observed in the $120 \mathrm{kHz}$ echosounder data. The lower color bar applies to both bottom panels. 
interface between strong and weak backscattering. The spatial coverage of the acoustic data obtained in the HB mode [Fig. 3(d)] was significantly smaller than in the VB mode. In the image shown, the instrument tracked diagonally across the path of the wave train as the ship and internal wave moved relative to one another. As a result, the vertical extent of an HB image was controlled by the depths at which the instrument was sampling at a particular moment. Uncalibrated data from a hull-mounted $120 \mathrm{kHz}$ narrowband echosounder are shown in Fig. 3(c), coincident in time with the HB acoustic echogram in Fig. 3(d).

Acoustic spectra were calculated by incoherently averaging over a number of pings. To ensure that the acoustic sampling volumes in the $\mathrm{HB}$ and VB modes were similar, the exact number of pings and range bins averaged over varied so that the height of each sampling volume was fixed at $2 \mathrm{~m}$ and the width ranged from $\sim 15-25 \mathrm{~m}$. The $2 \mathrm{~m}$ vertical extent of the sampling volume was small enough to exclude anisotropy due to patches that were thin in the vertical dimension, as typical layers in this study were observed to have vertical extent larger than $2 \mathrm{~m}$. The geometries of these sampling volumes depend on the orientation of the instrument and its movement relative to the internal wave train (Fig. 2; see Leong, 2009, for full details). A typical spectrum is shown in Fig. 4(a).

Spectra were calculated from $\mathrm{HB}$ and $\mathrm{VB}$ casts over eight and nine different wave trains, respectively, and classified according to their shape. To quantify the spectrum shape, two degree polynomials, which allow for curvature in the spectral shape, were fit independently to each of the lower frequency portion of the spectrum (LOW/MID band) and the higher frequency portion of the spectrum $(\mathrm{HL} / \mathrm{HH}$ band) [Fig. 4(b)]. The spectra were separated in this manner because each band pair shares similarities in transducer noise levels and ping rate. If there were obvious irregularities in spectra due to instrumental differences, these spectra were identified and discarded. The four frequency bands were not fit individually because it was easier to ascertain the overall spectral shape over a broader range of frequencies, particularly since the edges of each frequency band tended to deviate from the general shape. Fits over a larger fraction of the total spectrum were also better at establishing whether spectral features, such as a slope, were consistent.

The total frequency dependent error of each spectral fit was calculated by combining the estimated standard errors of the fit $(\delta F)$ with the standard deviations from the mean spectra $(\delta \bar{S})$, at each frequency, according to

$$
\text { total error of spectrum fit }=F \sqrt{\left(\frac{\delta F}{F}\right)^{2}+\left(\frac{\delta \bar{S}}{\bar{S}}\right)^{2}},
$$

where $\delta F$ measures both the uncertainty in estimating the polynomial, and the additional uncertainty in predicting a new observation. Once the fits were performed, the difference between the endpoints of each fitted curve was calculated. If this difference exceeded the average size of the total fit error bars, then it represented a significant rise or drop in spectral level and was classified as a "rise" or "drop" for that portion of the spectrum. For example, in Fig. 4, the lower frequency portion of the spectrum showed a significant drop in spectral level because the difference between the scattering levels at the endpoints exceeded the average errors for the fit. The higher frequency portion of the spectrum was considered flat because the scattering levels of the endpoints were within the fit error bars. Nine overall spectral shapes were defined using the combinations of flat, drop, or rise from the two fitted sections of spectra.

For each acoustic spectrum, the spectral shape was assigned by performing the fits described above on 100 bootstrap samples. For each bootstrap sample, the high- and lowfrequency portions of the spectrum were each randomly resampled. The spectrum fitting procedure was applied to determine the shape of each resampled spectrum portion and the overall shape of the resampled spectrum. The most common shape (mode) from the 100 bootstrap samples was assigned as the spectral shape, and the uncertainty of the assigned shape was calculated as the fraction of bootstrap sampled spectra that were not the mode shape.

\section{B. Microstructure observations}

Direct measurements of turbulent microstructure were made with the free-falling microstructure profiler Chameleon
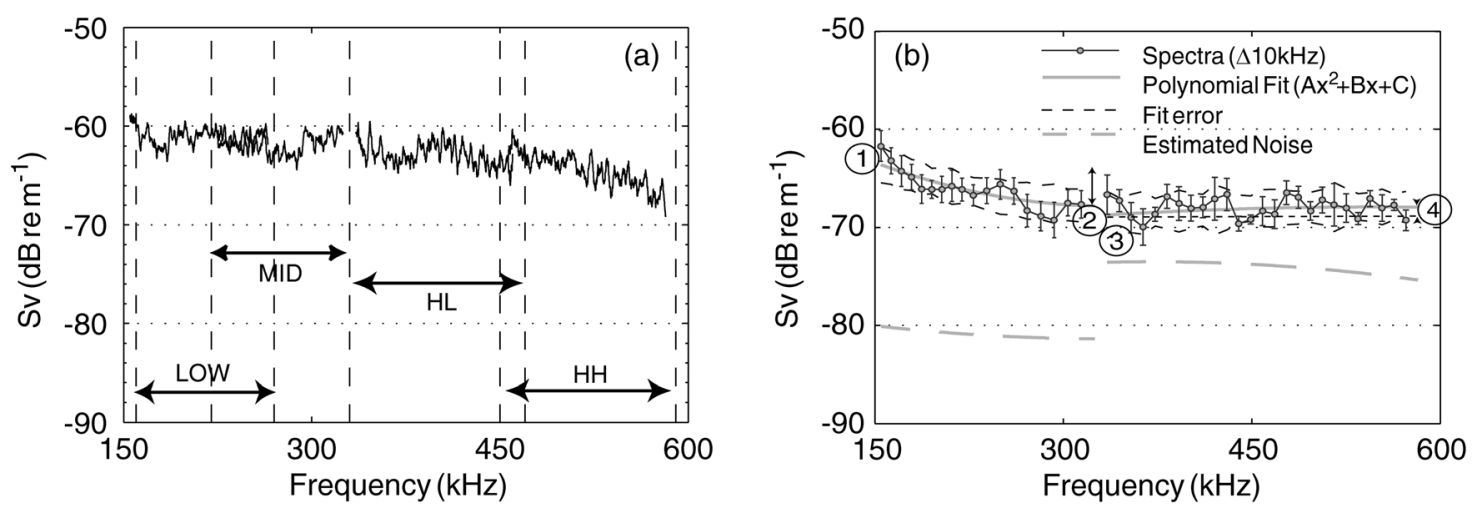

FIG. 4. (a) A HB sampled broadband acoustic spectrum from the pycnocline, labeled with the frequency band ranges. An average of 10 pings, each over a $3 \mathrm{~m}$ instrument range, was taken. (b) Method of determining spectral shape. The lowest and highest frequency sections of spectra were each fitted to a second order polynomial and the difference between the endpoints of each fit were calculated. The difference between endpoints are shown between arrowheads. The difference between endpoints (1) and (2) shows a significant drop in spectral level, while the difference between endpoints (3) and (4) is small and describes a relatively flat spectrum. Note that the vertical scale for $S_{v}$ includes the typical range of observed scattering and instrument noise levels and was applied to all subsequent figures for ease of comparison. 
(Moum et al., 1995; Tang et al., 2007), which was released off the stern of the ship as each solitary wave train was tracked. The profiler was deployed continuously, taking one profile every $40 \mathrm{~s}$ to $2 \mathrm{~min}$. Using data collected with Chameleon (Fig. 2), it was possible to estimate the turbulent kinetic energy dissipation rate $(\epsilon)$ and temperature variance dissipation rate $\left(\chi_{T}\right)$ (Moum et al., 1995; Nash et al., 1999). The salinity variance dissipation rate $\left(\chi_{S}\right)$ was estimated according to (Gregg, 1984; Moum 1996)

$$
\chi_{S}=\frac{(d S / d z)^{2}}{(d T / d z)^{2}} \chi_{T} .
$$

Outside the pycnocline, where temperature and salinity gradients are low, estimates of $\chi_{S}$ have greater uncertainty.

\section{Biological sampling}

Four net tows, labeled MOC(2,3,4,5) (Fig. 1), were performed during daylight hours using the Multiple Opening/ Closing Net and Environmental Sampling System (MOCNESS) (Wiebe et al., 1985). The MOCNESS consists of a maximum of ten nets that can be remotely opened and closed at selected intervals in order to sample a specific depth range and provided information on the abundance and species composition of zooplankton in the shelf waters.

Predicted acoustic scattering contributions from the zooplankton in these samples are shown in Fig. 5. The top row shows the sampling depth for each net and the middle row compares the mean backscattering contributions in the $\mathrm{HL} / \mathrm{HH}$ frequency bands for the dominant scatterers. Forward calculations based on scattering models described in Lavery et al.
(2007) show that copepods, pteropods, amphipods, and chaetognaths are all candidates to contribute to observed spectra because they can potentially scatter above the broadband instrument noise levels, mainly in the $\mathrm{HL} / \mathrm{HH}$ band. Other animals identified in the net tows include gelatinous medusae and salps, elastic-shelled bivalves and foraminifera, and fluid-like cladocerans, clione, crustaceans, decapods, ostracods, polychaetes, and gas-bearing siphonophores. The combined predicted scattering strength from these other taxa also exceeded instrument noise levels for some nets at some locations. The bottom row of Fig. 5 shows the total volume scattering strength from all animals in each net. An attempt was made to identify which nets were collected in the pycnocline (solid lines), that is, in the active region of the internal waves, though there was quite a bit of uncertainty because coincident Chameleon microstructure observations of the pycnocline depth were only made during MOC 4 and 5. For MOC 2 and 3, the pycnocline depth was based on the nearest microstructure data (collected a few hours apart and at a different location).

\section{CLASSIFICATION OF ACOUSTIC SPECTRA}

Acoustic spectra were sorted into one of three categories based on which source of scattering best explains their shape. Figure 6 shows examples of the seven spectral shapes commonly observed in the data set.

\section{A. Zooplankton-like spectra}

Zooplankton net tows provided rough estimates of species composition, abundance, and size, and were used to identify possible scattering contributions from zooplankton.
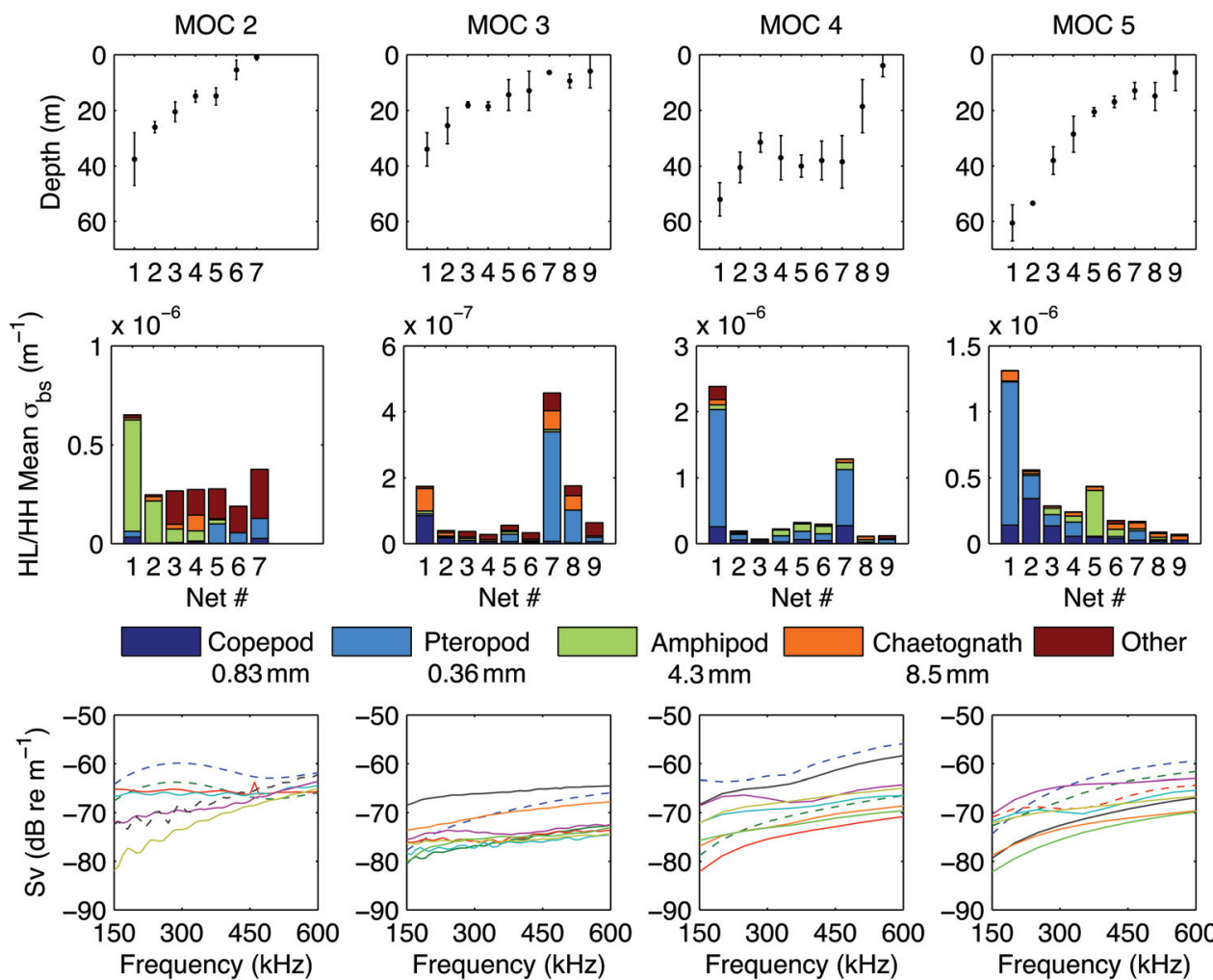
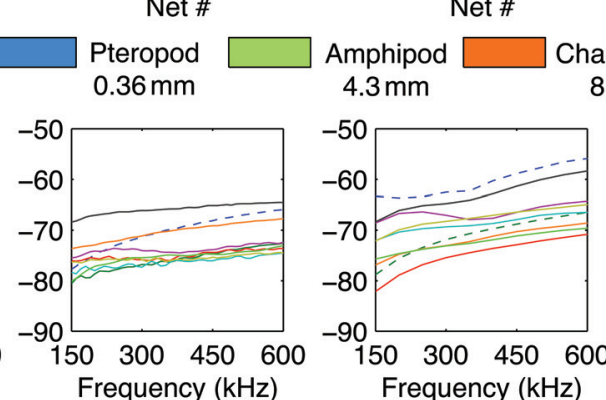

\section{$8.5 \mathrm{~mm}$}

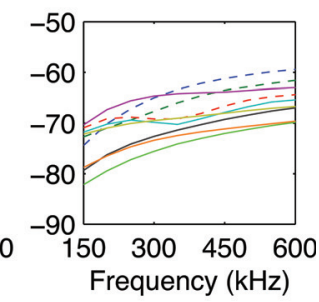

- Net $1-$ Net $2-$ Net $3-$ Net $4-$ Net 5
Net 6
FIG. 5. Depth ranges for the MOCNESS net tows are shown in the top row. The middle row shows mean backscattering cross-section, over the two highest frequency ranges $(\mathrm{HL} / \mathrm{HH})$ for each of the dominant scatterers. The mean lengths of each animal are given in the associated legend. The bottom row shows $S_{v}$ of all animals present in each net. Spectra calculated from nets outside the pycnocline are indicated as dashed lines. 

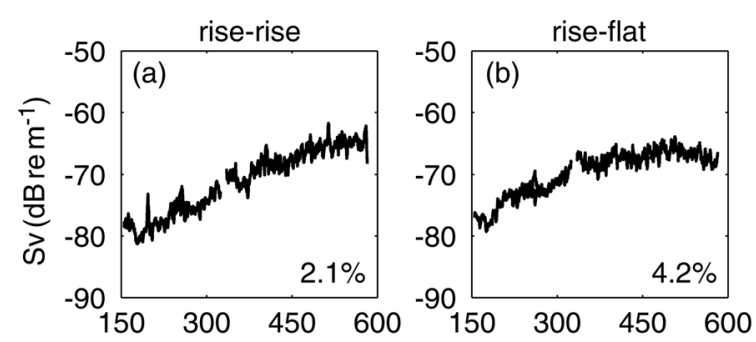

zooplankton
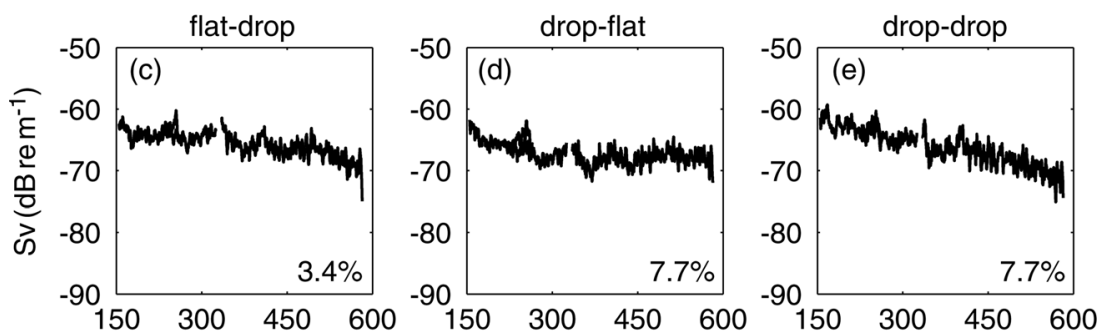

turbulence
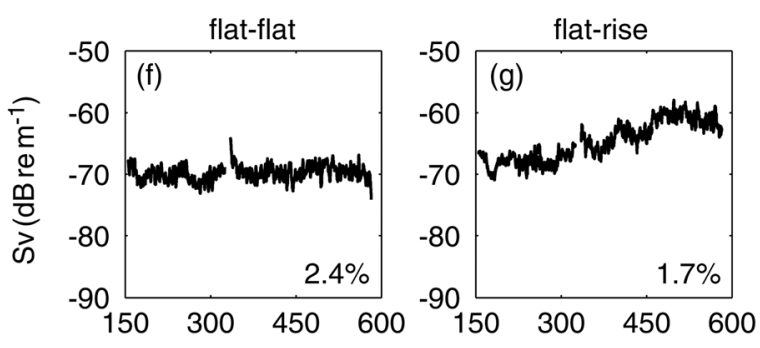

Frequency $(\mathrm{kHz})$

FIG. 6. Examples of spectral shapes. (a) and (b) show examples of small zooplankton spectra, (c)-(e) show examples of turbulence spectra, and (f) and (g) show examples of ambiguous spectra. The average classification uncertainty for each shape is given in the lower right of each panel. The combinations "rise-drop" and "drop-rise" were rarely observed and are not shown.
Rayleigh scattering from copepods, pteropods, and chaetognaths was predicted throughout all or most of the frequency range of the broadband acoustic instrument for almost all of the nets collected in the region (Fig. 5). As a result, the majority of zooplankton spectra increase steeply with increasing frequency. In a few of the nets, the predicted scattering was dominated by organisms with relatively flat spectra (amphipods, some large pteropods, and combinations of other weakly scattering organisms), which can contribute to observed scattering in both low and high frequency bands. These were difficult to identify conclusively as zooplankton based on spectral shape alone (see Fig. 7).

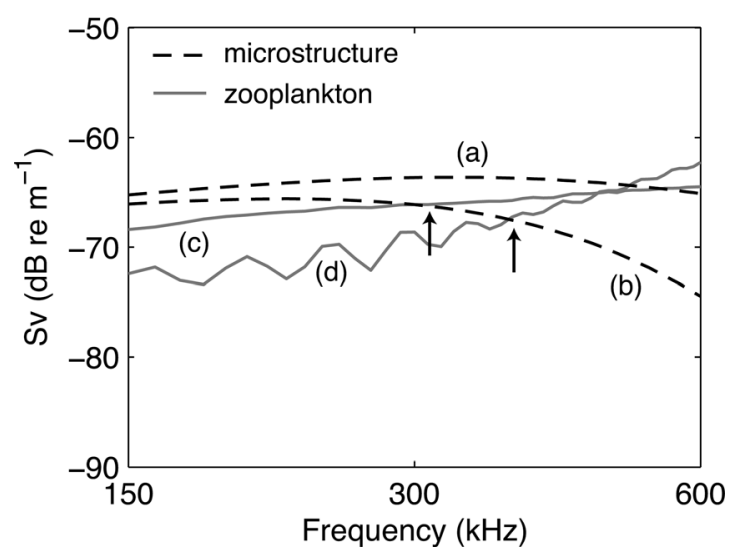

FIG. 7. Predicted spectra calculated from Chameleon microstructure data (a) and (b), and MOCNESS zooplankton data (c) and (d). The classification of flat spectra is ambiguous because it can originate from both turbulence (a), or zooplankton (c). Zooplankton backscattering at high frequencies (c) and (d), can also obscure the spectral roll-off of turbulence (b), beginning at the arrow positions, producing spectra that appear ambiguous in origin.
The spectra in Figs. 6(a) and 6(b) were identified as zooplankton spectra because they show the frequency dependence of discrete scatterers in the Rayleigh regime or near the Rayleigh-to-geometric transition point. This includes spectra which rise sharply at all frequencies, as in Fig. 6(a), or rise initially and subsequently flatten at higher frequencies, as in Fig. 6(b).

\section{B. Turbulence-like spectra}

Sound is scattered from wavelength-scale variations in the acoustic impedance created by turbulence. In seawater, acoustic impedance depends on temperature and salinity, and the backscattering cross-section can be expressed in terms of the turbulent kinetic energy dissipation rate $\epsilon$, temperature variance dissipation rate $\chi_{T}$, and salinity variance dissipation rate $\chi_{S}$ in the following way (Ross et al., 2004):

$$
\begin{aligned}
\sigma_{\mathrm{bs}}= & \frac{q \kappa}{16}\left(\frac{\nu}{\epsilon}\right)^{1 / 2}\left(A^{2} \chi_{T} e^{-4 q\left(\kappa / k_{B T}\right)^{2}}+B^{2} \chi_{S} e^{-4 q\left(\kappa / k_{B S}\right)^{2}}\right. \\
& \left.+2 A B\left(\chi_{T} \chi_{S}\right)^{1 / 2} e^{-4 q\left(\kappa / k_{B T S}\right)^{2}}\right)
\end{aligned}
$$

where $q$ is an empirical constant usually taken to be 3.7 (Oakey, 1982), $\nu$ is the kinematic viscosity, $A=a-\alpha$, $B=b+\beta, a$ and $b$ are the fractional changes in sound speed due to temperature and salinity changes, $\alpha$ and $\beta$ are the coefficients of thermal expansion and saline contraction, and $k_{B(T, S, T S)}$ are the Batchelor wavenumbers for temperature spectra, salinity spectra, or temperature-salinity co-spectra; $k_{B(T, S)}=\left(\epsilon /\left(\nu D_{(T, S)}^{2}\right)\right)^{1 / 4}$, where $D_{(T, S)}$ is the diffusivity of heat or salinity, and $k_{B T S}=\left(4 \epsilon / \nu\left(D_{T}+D_{S}\right)^{2}\right)^{1 / 4}$. The 
acoustic wavenumber, $\kappa$, is directly proportional to the acoustic frequency, $f ; \kappa=2 \pi f / c$, where $\mathrm{c}$ is the local sound speed.

Turbulence spectra exhibit two characteristic spectral roll-offs associated with temperature and salinity dissipation. The positions of the spectral roll-offs are governed by the Batchelor wavenumbers in the exponential terms of Eq. (3). As a result, the magnitude of $\epsilon$ controls the frequencies at which the roll-offs occur.

The spectra in Figs. 6(c)-6(e) show a significant drop with increasing frequency over part or all of their spectrum, which is characteristic of scattering from turbulence (Warren et al., 2003; Lavery et al., 2010a). For example, Fig. 6(c) shows an initially flat spectrum that rolls-off at higher frequencies, which likely corresponds to the roll-off of a temperature dominated turbulent scattering spectra with a high energy dissipation rate. Figure 6(d) shows an immediate drop at low frequency and then flattens out at high frequencies, which likely corresponds to temperature dominated turbulent microstructure with relatively strong salinity microstructure present, but could also indicate that turbulence only dominates the first half of the spectrum. Figure 6(e) shows a significant drop across all frequencies, which likely corresponds to temperature dominated spectra with a low energy dissipation rate. While scattering from the gas-inclusions of siphonophores can produce a decreasing spectrum from low to high frequencies (Lavery et al., 2007), the net tow data suggest that most scattering from siphonophores would be below the noise level of all transducers (Fig. 5).

\section{Spectra of ambiguous origin}

The spectral shapes shown in the bottom row of Fig. 6, and the rarely observed rise-drop and drop-rise shapes, were classified as ambiguous because it was impossible to determine, with the bandwidth available, the source of the backscattering.

Flat spectra can arise if the position of a turbulent spectral roll-off occurs at frequencies that are outside the frequency range of the broadband instrument [e.g., spectrum (a) in Fig. 7]. The total backscattering from zooplankton can also produce a flat spectrum [e.g., spectrum (c) in Fig. 7 calculated from MOC 3]. Figure 7 also shows how spectra of ambiguous origins may arise from a combination of backscattering from zooplankton and turbulence. High-frequency zooplankton backscattering may obscure a turbulent spectral roll-off, resulting in a generally flat spectrum [e.g. combination of spectra (b) and (c)], or an initially flat spectrum that rises sharply at high frequencies [e.g. combination of spectra (b) and (d)].

\section{ANISOTROPY IN THE SPECTRAL CLASSIFICATIONS}

The isotropy of the backscattering was examined in three separate regions of the internal wave: above, at, and below the pycnocline. First, the acoustic spectra were sorted into these three distinct regions, with the pycnocline depth defined using density contours calculated from the Chameleon microstructure data [Fig. 3(a)]. Since this choice of pycnocline was subject to temporal aliasing due to the low frequency of the microstructure profiles relative to the acoustic sampling rate,
TABLE I. Number of spectra analyzed in both sampling orientations, in each region relative to the pycnocline. The columns list the number of spectra of each spectral classification (ambiguous, zooplankton, turbulence), the number of successful acoustic spectral inversions performed for microstructure parameters, and the total number of spectra above noise levels.

Ambiguous Zooplankton Turbulence Inversions Total

\begin{tabular}{lccccc}
\hline $\begin{array}{l}\text { Above } \\
\text { pycnocline, HB }\end{array}$ & 184 & 1 & 273 & 238 & 458 \\
$\begin{array}{l}\text { Above } \\
\text { pycnocline, VB }\end{array}$ & 887 & 21 & 83 & 44 & 991 \\
$\begin{array}{l}\text { At } \\
\text { pycnocline, HB }\end{array}$ & 380 & 88 & 652 & 533 & 1120 \\
$\begin{array}{l}\text { At } \\
\text { pycnocline, VB }\end{array}$ & 8127 & 800 & 1824 & 382 & 10751 \\
$\begin{array}{l}\text { Below } \\
\text { pycnocline, HB }\end{array}$ & 53 & 86 & 14 & 5 & 153 \\
$\begin{array}{l}\text { Below } \\
\text { pycnocline, VB }\end{array}$ & 1117 & 4621 & 93 & 2 & 5831 \\
\hline \hline
\end{tabular}

the position of the pycnocline may be uncertain by a few meters. The total number of spectra (above noise levels) collected in each region is listed in Table I. For each of the three regions, Fig. 8 shows, and Table I also lists, the distribution of spectral classifications measured in each sampling orientation.

Below the pycnocline, non-turbulent scattering dominated the acoustic returns in both $\mathrm{HB}$ and VB modes. Little scattering was predicted from turbulent microstructure in the weak temperature and salinity gradients below the pycnocline, and biological scatters should dominate the scattering in this region. At the pycnocline, where scattering from turbulent microstructure was expected to be strongest, the majority of HB spectra were classified as turbulence-like (Fig. 8). In contrast, VB spectra from this region were mainly ambiguous. The distribution of scattering spectra classifications above the pycnocline and at the pycnocline were similarly distinct between $\mathrm{HB}$ and $\mathrm{VB}$, indicating

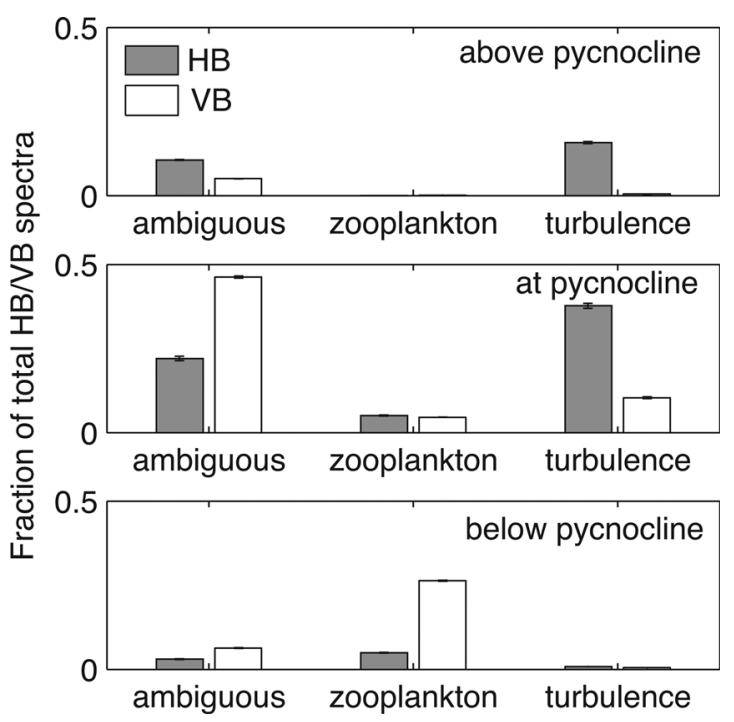

FIG. 8. Distribution of scattering spectra classifications in each observed region of the water column, for each sampling orientation. Each bar was normalized to the total number of spectra calculated in its corresponding sampling orientation. Error bars show the uncertainties obtained from bootstrap sampling, but were very small and not visible in most cases. 
scattering anisotropy. The potential contributions to this scattering anisotropy by zooplankton and turbulent microstructure sources are investigated in the following sections.

\section{ZOOPLANKTON ORIENTATION}

The scattering anisotropy observed at the pycnocline may be due to anisotropy in zooplankton scattering if longaspect-ratio zooplankton, such as euphausiids, were oriented preferentially horizontally (see references in Sec. I). Generally, horizontally orientated elongated zooplankton will scatter more strongly in VB mode relative to $\mathrm{HB}$ mode. If the mean scattering strength of the zooplankton was stronger in the VB mode, this would obscure the identification of turbulence spectra in VB mode (Fig. 7). More spectra would then be identified as ambiguous in the VB mode, as was observed. However, the daytime net tows conducted suggest that animals with small aspect ratios dominated much of the scattering (Fig. 5), for which any orientation effects are predicted to be small and do not explain the observed scattering anisotropy.

The species present in the pycnocline may be different during the day and night, however, and there was evidence of diel vertical migration of zooplankton (Lavery et al., 2010a), where a strong dense scattering layer appeared at night at and above the pycnocline, but was not generally present during daytime hours. Acoustic data collected in the daytime was contrasted with that collected at night, to explore whether day and night distributions of zooplankton may be responsible for the observed anisotropy (Fig. 9). The percent change in the number of nighttime spectra relative to daytime spectra was calculated for each classification group. For both HB and VB data, there was a $300 \%$ or more increase in the number of spectra classified as zooplankton-like during the night, which was consistent with the observation of diel vertical migration. However, there were very small differences between the HB and VB spectral classification, suggesting that day and night differences did not affect scattering anisotropy.

\section{ACOUSTIC INVERSIONS FOR TURBULENCE PARAMETERS}

Turbulence anisotropy is another potential contributor to the observed overall scattering anisotropy. In this section

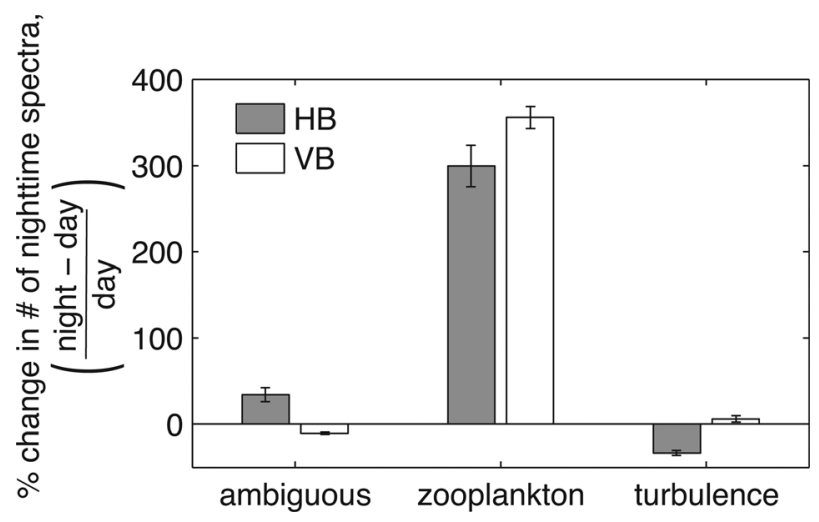

FIG. 9. Percent change in the number of nighttime spectra relative to the number of daytime spectra for each spectral classification and both sampling orientations, in the pycnocline. Error bars show only the uncertainties obtained from bootstrap sampling. we explore how acoustically estimated turbulence parameters are affected by turbulence anisotropy.

In the Goodman (1990) model for sound scattering from turbulence, the author suggested a method for parameterizing anisotropy, which for turbulence is expected to decrease the vertical dimension of microstructure relative to the horizontal due to stratification, by scaling the vertical wavenumber. The anisotropy parameter, $\zeta(0<\zeta \leq 1)$, equivalent to the parameter $\alpha$ in Goodman (1990), represents the ratio between the horizontal and vertical wavenumbers such that $\zeta$ scales the vertical wavenumber to its isotropic value and equivalent to the horizontal wavenumber. Requiring $\zeta$ less than unity assumes that strong stratification limits vertical fluid motion. Extending Goodman's (1990) derivation, we assumed that the vertical wavenumber was scaled for anisotropy and that the three-dimensional temperature and salinity spectra followed the Batchelor form (Batchelor, 1959). We found that backscattering from anisotropic turbulence should follow the same functional form as for isotropic turbulence, that is Eq. (3), with the caveat that the apparent dissipation rates will be different depending on the angle of insonification. For horizontally and vertically sampled spectra, the apparent magnitudes of $\chi_{T, S}$ and $\epsilon$ were related as follows:

$$
\begin{gathered}
\epsilon^{V B}=\frac{\epsilon^{H B}}{\zeta^{4}}, \\
\chi_{T, S}^{V B}=\frac{\chi_{T, S}^{H B}}{\zeta^{5}},
\end{gathered}
$$

which predict that VB values will be greater than $\mathrm{HB}$ values for anisotropic turbulence with $\zeta<1$. Acoustically, the result would be stronger scattering in VB mode relative to $\mathrm{HB}$ mode and also the spectral roll-off would occur at higher acoustic frequencies in the VB relative to the HB. This was consistent with the observation that ambiguous spectra were observed much more frequently in VB mode relative to $\mathrm{HB}$ mode; anisotropy would shift the spectral roll-off of the VB spectra, which in HB mode occurred within the band of the broadband acoustic instrument, to frequencies outside the range of observation.

The predominance of ambiguous spectra in VB mode could also be a consequence of anisotropy in the zooplankton scattering. Equations (4) and (5) were therefore used to evaluate the level of turbulence anisotropy by performing an inversion on the spectra that were classified as turbulence. Not all spectra that were classified as turbulence in Sec. IV could be inverted, however, because the position of the spectral roll-off must be known in order to accurately estimate $\epsilon$. Examples of microstructure scattering spectra observed by the broadband instrument in this study can be found in Figs. 5-8 of Lavery et al. (2010b) For all spectra that showed a clear turbulent roll-off (i.e., those with a "flat-drop" shape; listed in Table I), the turbulence model of Eq. (3) was fitted using nonlinear least squares regression to estimate the turbulence parameters. The inversion was performed in linear space, and involved two free parameters $\epsilon$ and $\chi_{T}$, together with a fixed ratio for $A^{2} \chi_{T} / B^{2} \chi_{S}$ that was determined using Chameleon microstructure data from the pycnocline. This 

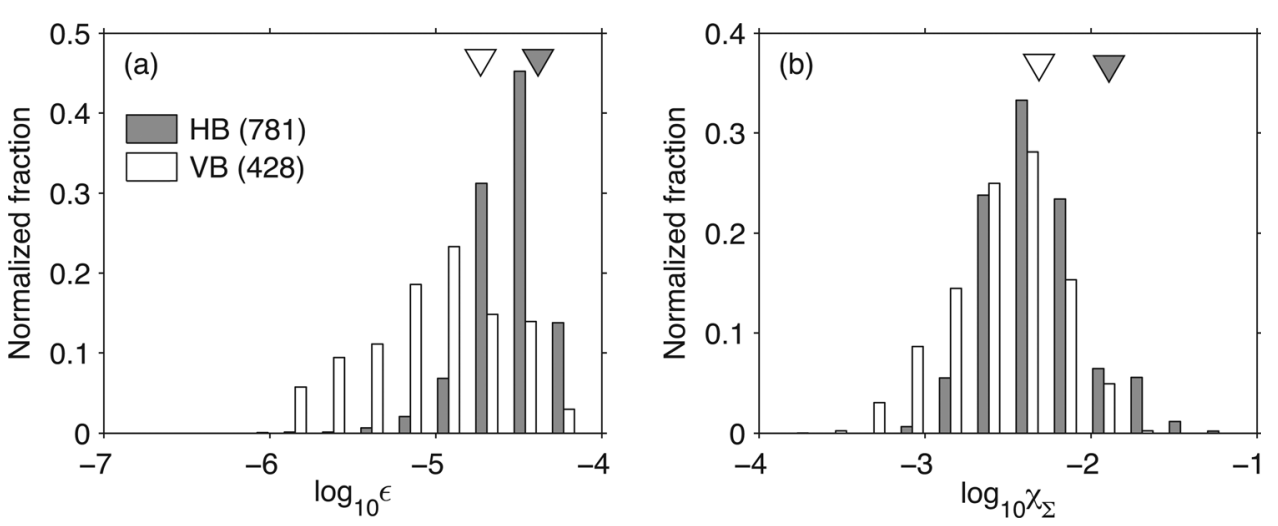

FIG. 10. Distribution of turbulence parameters (a) $\epsilon$ and (b) $\chi_{\Sigma}$, estimated from acoustic spectral inversions for each sampling orientation. The fraction of total counts is given for each bin and includes the uncertainties from each estimate of $\epsilon$ and $\chi_{\Sigma}$. Inverted triangles show the mean values, with the standard errors too small to be shown. The total number of spectra is given in brackets in the legend. ratio must be fixed because of the limited frequency range of the broadband acoustic instrument. Since $\chi_{T}$ and $\chi_{S}$ must be linked, their combined contribution to scattering before the first spectral roll-off [Eq. (3)] was more robustly represented by the parameter

$$
\chi_{\Sigma}=\chi_{T}+\frac{B^{2}}{A^{2}} \chi_{S}+\frac{2 B}{A}\left(\chi_{T} \chi_{S}\right)^{1 / 2},
$$

which has units of temperature variance dissipation rate.

Histograms of the acoustically inverted turbulence parameters were constructed using the expected values and uncertainties for each estimated turbulence parameter (Fig. 10). The expected probability that each estimated $\epsilon$ or $\chi_{\Sigma}$ lay within a certain range (or histogram bin) was calculated, and the probabilities for each range of values were then summed. These distributions of the apparent magnitudes of $\chi_{\Sigma}$ and $\epsilon$ estimated from HB and VB spectra could then be compared and used to estimate $\zeta$. The VB versus HB ratios for the $\epsilon$ and $\chi_{\Sigma}$ distribution means were $\sim 0.45$ and $\sim 0.38$, respectively. These shifts were in the opposite direction to what is expected from Eqs. (4) and (5) with $\zeta<1$. Ignoring for the moment that $\zeta$ is expected to be less than 1 , the magnitudes of the shifts correspond to an anisotropy parameter of $\zeta=1.2$. This unconventional value for the anisotropy parameter likely arose because turbulence statistics were not based on true representative samples of scattering from both directions, due to the limited bandwidth available. Limited bandwidth altered the distributions of inverted turbulence parameters as compared to directly measured turbulence parameters (Leong, 2009; Lavery et al., 2010a,b). If anisotropy was present, it is possible that the HB and VB distributions were altered in different ways. Without resolving the full spectrum of scattering, it is unknown whether the VB turbulence spectra identified were biased toward lower turbulence parameter values or generally how many spectra were misidentified as turbulence, zooplankton, or ambiguous.

\section{CONCLUSIONS}

In this study we report on the observation of significant scattering anisotropy as measured by high-frequency broadband $(120-600 \mathrm{kHz})$ acoustic scattering in the presence of internal waves, where either turbulent microstructure or zooplankton (or both) are expected to be dominant sources of acoustic scattering. There have been few previous high-frequency measurements of scattering that allow anisotropy in the presence of zooplankton and microstructure to be assessed, and, in particular, there have been no previous measurements of broadband acoustic scattering that address the question of scattering anisotropy. Yet it is important to address the issue of scattering anisotropy as this can potentially interfere with the accurate interpretation and classification of the scattering returns.

The source of the scattering anisotropy measured in this study remains unclear. This scattering anisotropy may be a result of elongated zooplankton that preferentially orientate horizontally, although this is not the conclusion of the modeling results based on zooplankton net tows collected in the general area of study (not simultaneous collections). Alternatively it could be a result of scattering from anisotropic turbulent microstructure. However, acoustic inferences of dissipation rates of turbulent kinetic energy and temperature variance are at odds with predictions based on a model for scattering from anisotropic turbulent microstructure. Thus, although there is clear evidence for high-frequency scattering anisotropy in the vicinity of internal waves, the source of scattering could not be determined in this study.

While the analysis performed here would not have been possible without a broadband system, the largest uncertainty in identifying scattering sources was related to the limited bandwidth of the system. Many spectra could not be unambiguously classified as due to turbulence or zooplankton given the available bandwidth. Increasing the lower end of the frequency band to approximately $50 \mathrm{kHz}$ would have made possible the identification of the Rayleigh-to-geometric scattering transition for larger zooplankton (such as euphausiids), and increasing the upper end of the available bandwidth to $1-2 \mathrm{MHz}$, might have allowed a more robust identification of the roll-off in the temperature spectrum, and ideally even the roll-off in the salinity dissipation spectrum. In conclusion, although the source of scattering anisotropy could not be conclusively identified, this study indicates the importance of considering the effects of scattering anisotropy in the vicinity of internal waves, and the great potential of these broadband techniques in measuring, assessing, classifying, and interpreting acoustic scattering anisotropy.

Batchelor, G. (1959). "Small-scale variation of convected quantities like temperature in turbulent fluid,” J. Fluid Mech. 5, 113-133.

Benfield, M. C., Davis, C. S., and Gallager, S. M. (2000). "Estimating the in-situ orientation of calanus finmarchicus on Georges Bank using the video plankton recorder," Plankton Biol. Ecol. 47, 69-72. 
Benoit-Bird, K., and Au, W. (2009). "Phonation behavior of cooperatively foraging spinner dolphins," J. Acoust. Soc. Am. 125, 539-546.

Bertrand, A., Gerlotto, F., Bertrand, S., Gutierrez, M., Alza, L., Chipollini, A., Diaz, E., Espinoza, P., Ledesma, J., Quesquen, R., Peraltilla, S., and Chavez, F. (2008). "Schooling behaviour and environmental forcing in relation to anchoveta distribution: An analysis across multiple spatial scales," Prog. Oceanogr. 79, 264-267.

Chu, D., Foote, K. G., and Stanton, T. K. (1993). "Further analysis of target strength measurements of Antarctic krill at 38 and $120 \mathrm{kHz}$ : Comparison with deformed cylinder model and inference of orientation distribution," J. Acoust. Soc. Am. 93, 2985-2988.

Chu, D., and Stanton, T. (1998). "Application of pulse compression techniques to broadband acoustic scattering by live individual zooplankton," J. Acoust. Soc. Am. 104, 39-55.

Gargett, A. (1984). "Local isotropy and decay of turbulence in a stratified fluid,” J. Fluid Mech. 144, 231-280.

Goodman, L. (1990). "Acoustic scattering from ocean microstructure," J. Geophys. Res. 95, 11557-11573.

Gregg, M. (1984). "Entropy generation in the ocean by small-scale mixing," J. Phys. Oceanogr. 14, 688-711.

Haury, L., Wiebe, P., Orr, M., and Briscoe, M. (1983). "Tidally generated high-frequency internal wave-packets and their effects on plankton in Massachusetts Bay,” J. Mar. Res. 41, 65-112.

Holliday, D., and Pieper, R. (1995). "Bioacoustical oceanography at high frequencies,” ICES J. Mar. Sci. 52, 270-296.

Jaffe, J., Ohman, M., and De Robertis, A. (1998). "OASIS in the sea: Measurement of the acoustic reflectivity of zooplankton with concurrent optical imaging," Deep-Sea Res., Part II 45, 1239-1253.

Kolmogorov, A. (1941). "The local structure of turbulence in an incompressible viscous fluid for very large Reynolds number," C. R. Acad. Sci. USSR 30, 301-305.

Lavery, A., Chu, D., and Moum, J. (2010a). "Measurements of acoustic scattering from zooplankton and oceanic microstructure using a broadband echosounder," ICES J. Mar. Sci. 67, 379-394.

Lavery, A., Chu, D., and Moum, J. (2010b). "Observations of broadband acoustic backscattering from nonlinear internal waves: Assessing the contributions from microstructure and zooplankton," IEEE J. Ocean. Eng. 35, 695-709.

Lavery, A., Schmitt, R., and Stanton, T. (2003). "High-frequency acoustic scattering from turbulent oceanic microstructure: The importance of density fluctuations," J. Acoust. Soc. Am. 114, 2685-2697.

Lavery, A., Wiebe, P., Stanton, T., Lawson, G., Benfield, M., and Copley, N. (2007). "Determining dominant scatterers of sound in mixed zooplankton populations," J. Acoust. Soc. Am. 122, 3304-3326.

Lawson, G., Wiebe, P., Ashjian, C., Chu, D., and Stanton, T. (2006). "Improved parameterization of Antarctic krill target strength models," J. Acoust. Soc. Am. 119, 232-242.

Leong, D. (2009). "Assessing the isotropy of ocean turbulence using broadband acoustics," Master's thesis, Dalhousie University.

Martin Traykovski, L. (1998). "Effect of orientation on broadband acoustic scattering of Antarctic krill Euphausia superba: Implications for inverting zooplankton spectral acoustic signatures for angle of orientation," J. Acoust. Soc. Am. 104, 2121-2135.

McGehee, D., O'Driscoll, R., and Martin Traykovski, L. (1998). "Effects of orientation on acoustic scattering from Antarctic krill at $120 \mathrm{khz}$," DeepSea Res., Part II 45, 1273-1294.

Midttun, L. (1984). "Fish and other organisms as acoustic targets," Rapports et Procès-Verbaux des Réunions du Conseil International pour l'Exploration de la Mer 184, 25-33.

Monin, A., and Yaglom, A. (1965). Statistical Fluid Mechanics II (MIT Press, Cambridge), pp. 1-896.

Moum, J. (1996). "Efficiency of mixing in the main thermocline," J. Geophys. Res. 101, 12057-12069.

Moum, J., Gregg, M., Lien, R., and Carr, M. (1995). "Comparison of turbulence kinetic energy dissipation rate estimates from two ocean microstructure profilers," J. Atmos. Ocean. Technol. 12, 345-366.

Moum, J., and Rippeth, T. (2009). "Do observations adequately resolve the natural variability of oceanic turbulence?,” J. Mar. Syst. 77, 409-417.

Moum, J. N., Farmer, D. M., Smyth, W. D., Armi, L., and Vagle, S. (2003). "Structure and generation of turbulence at interfaces strained by internal solitary waves propagating shoreward over the continental shelf," J. Phys. Oceanogr. 33, 2093-2112.
Nash, J. D., Caldwell, D. R., Zelman, M. J., and Moum, J. N. (1999). “A thermocouple probe for high-speed temperature measurement in the ocean," J. Atmos. Ocean. Technol. 16, 1474-1482.

Naylor, E. (2006). "Orientation and navigation in coastal and estuarine zooplankton,” Mar. Freshwater Behav. Physiol. 39, 13-24.

Oakey, N. (1982). "Determination of rate of dissipation of turbulent energy from simultaneous temperature and velocity shear microstructure measurements," J. Phys. Oceanogr. 12, 256-271.

Oeschger, J., and Goodman, L. (2003). "Acoustic scattering by a thermally driven buoyant plume revisited," J. Acoust. Soc. Am. 113, 1353-1367.

Roberts, P. L. D., and Jaffe, J. S. (2008). "Classification of live, untethered zooplankton from observations of multiple-angle acoustic scatter," J. Acoust. Soc. Am. 124, 796-802.

Ross, T., Garrett, C., and Lueck, R. (2004). "On the turbulent co-spectrum of two scalars and its effect on acoustic scattering from oceanic turbulence," J. Fluid. Mech. 514, 107-119.

Ross, T., and Lueck, R. (2005). "Estimating turbulent dissipation rates from acoustic backscatter,” Deep-Sea Res., Part I 52, 2353-2365.

Sameoto, D. D. (1980). "Quantitative measurements of euphausiids using a $120-\mathrm{kHz}$ sounder and their in situ orientation," Can. J. Fish. Aquat. Sci. 37, 693-702.

Sandstrom, H., and Oakey, N. S. (1995). "Dissipation in internal tides and solitary waves," J. Phys. Oceanogr. 25, 604-614.

Seim, H. (1999). "Acoustic backscatter from salinity microstructure," J. Atmos. Ocean. Technol. 16, 1491-1498.

Simard, Y., and Sourisseau, M. (2009). "Diel changes in acoustic and catch estimates of krill biomass," ICES J. Mar. Sci. 66, 1318-1325.

Simmonds, J., and MacLennan, D. (2005). Fisheries Acoustics, Theory and Practice, Vol. 10 of Fish and Aquatic Resources Series (Blackwell, Oxford), Chap. 7, pp. 266-276.

Stanton, T., and Chu, D. (2000). "Review and recommendations for modeling of acoustics scattering by fluid-like elongated zooplankton: Euphausiids and copepods," ICES J. Mar. Sci. 57, 793-807.

Stanton, T., and Chu, D. (2008). "Calibration of broadband active acoustics systems using a single standard spherical target," J. Acoust. Soc. Am. 124, $128-136$.

Stanton, T. K., Chu, D., Jech, J. M., and Irish, J. D. (2010). "New broadband methods for resonance classification and high-resolution imagery of fish with swimbladders using a modified commercial broadband echosounder," ICES J. Mar. Sci. 67, 365-378.

Stanton, T. K., Wiebe, P. H., Chu, D., and Goodman, L. (1994). "Acoustic characterization and discrimination of marine zooplankton and turbulence," ICES J. Mar. Sci. 51, 469-479.

Sutor, M., Cowles, T., Peterson, W., and Lamb, J. (2005). "Comparison of acoustic and net sampling systems to determine patterns in zooplankton distribution," J. Geophys. Res. 110, C10S16.

Tang, D., Moum, J., Lynch, J., Abbott, P., Chapman, R., Dahl, P., Duda, T., Gawarkieweicz, G., Glenn, S., Goff, J., Graber, H., Kemp, J., Maffei, A., Nash, J., and Newhall, A. (2007). "Shallow water '06. A joint acoustic propagation/nonlinear internal wave physics experiment," Oceanogr. 20, 156-167.

Thorpe, S. (2005). The Turbulent Ocean (Cambridge University Press, Cambridge), pp. 1-439.

Trevorrow, M., Mackas, D. L., and Benfield, M. C. (2005). "Comparison of multifrequency acoustic and in situ measurements of zooplankton abundances in Knight Inlet, British Columbia," J. Acoust. Soc. Am. 117, $3574-3588$.

Warren, J., Stanton, T., McGehee, D., and Chu, D. (2002). "Effect of animal orientation of acoustic estimates of zooplankton properties," IEEE J. Ocean. Eng. 27, 130-138.

Warren, J., Stanton, T., Wiebe, P., and Seim, H. (2003). "Inference of biological and physical parameters in an internal wave using multiplefrequency, acoustic-scattering data," ICES J. Mar. Sci. 60, 1033-1046.

Wiebe, P., Chu, D., Kaartvedt, S., Hundt, A. W. Melle, E. O., and BattaLona, P. (2010). "The acoustic properties of Salpa thompsoni," ICES J. Mar. Sci. 67, 583-593.

Wiebe, P., Morton, A., Bradley, A., Backus, R., Craddock, J., V. Barber, Cowles, T., and Flierl, G. (1985). "New developments in the MOCNESS, an apparatus for sampling zooplankton and micronekton," Mar. Biol. (Berlin) 87, 313-323.

Wiebe, P., Stanton, T., Benfield, M., Mountain, D., and Greene, C. (1997). "High-frequency acoustic volume backscattering in the Georges Bank coastal region and its interpretation using scattering models," IEEE J. Ocean. Eng. 22, 445-464. 